\title{
Sensor System for Detection of Gunshot and the
}

\section{Localization System}

\author{
Aravindaguru I, Gowthami R, Sanjoydeb
}

\begin{abstract}
For quite a long time, the law requirement associations have progressively used sound location framework to recognize the potential gunfire in the open spots and in the woods. The killing of creature or natural life in timberland utilizing the weapon is expanding step by step; to identify the gunfire in the woods the sound identification framework has been proposed. The proposed frameworks have developed from straightforward receiver arrangements used to appraise area of gunfire inside insignificant feet of its genuine event. Moreover, however fundamental structures require little as far as programming or designing knowledge. At long last, the framework will be helpful to defeat the few issues like recognizing from which bearing the shot occurred and at what separation the discharge sound has happened. This examination work will assume a functioning job in the public arena now and later on, in extra applications also.
\end{abstract}

Keywords: LabVIEW, Gun shot, Microphone, Arduino, VISA

\section{INTRODUCTION}

Till now, there is no known measure that can effectively anticipate and avoid wrongdoing before it happens. In this way, law authorization offices ought to expand the effectiveness and exactness to identify the crime. The sooner law requirement faculty can have a nearness at the scene where a wrongdoing occurred, the sooner the territory may come back to a precise condition of wellbeing. Specialists can defuse hazardous circumstances, assemble proof, and manufacture a case used to catch those in charge of the wrongdoing.

Numerous variables limit the measure of impact law implementation organizations have on the overall population, and a portion of these elements present deterrents when taking a stab at auspicious and successful wrongdoing reaction. Spending plans and accessible subsidizing manage the measure of staff and hardware an organization can utilize, and enactment directs office control. Law authorization gatherings may mediate in a given circumstance, however just when strategically fit and legitimately allowed.

Revised Manuscript Received on August 14, 2019.

Aravindaguru I, Department of Electronics and Instrumentation Engineering, M.Kumarasamy College of Engineering, Karur, Tamil Nadu, India .

(Email: aravindagurui.eie@mkce.ac.in)

Gowthami R, Department of Electronics and Instrumentation Engineering, M.Kumarasamy College of Engineering, Karur, Tamil Nadu, India.

Sanjoydeb, Department of Electronics andCommunication Engineering, Bannari Amman Institute of Technology, Sathyamangalam, Tamil Nadu, India.
While positive expected consequences of this control incorporate safe and practical law authorization, tragically negative reactions additionally may emerge. Organizations might be understaffed or not well prepared, and might be limited from reacting as fast or as soundly as wanted. In light of that bargain, law authorization gatherings have started using the shot location and restriction framework.

\section{BASIC DESIGN}

Before amassing and testing an essential sound discovery framework, a general system must be plot. The framework to be planned in the accompanying advances will recognize sound of a specific arrangement of qualities, will infer a heading of starting point of the sound source, and will point the determined way.

In a genuine setting, such a framework would "initiate" when activated and would consequently point a hand-held gadget towards the decided sound source, with expectations of catching conceivably important proof to help examiners.

Remembering this desire, the framework will incorporate mouthpieces to catch sound, a PC to process the approaching sound and send directions, a microcontroller to get the directions and send relating voltages to the framework that is inserted with LABVIEW.

Alongside the hardware arranging, a methodology ought to be made for the framework programming. The two primary inquiries to answer are: by what means will the framework segregate discharge like sounds from different sounds? What's more, in what capacity will the framework decide the course of the sound source?

To separate shot like sounds from others, the hints of intrigue must be portrayed as far as quantifiable qualities. To the human ear, the most evident of these characteristics are the apparent commotion and brief length of the occasion. As per Michael and Lucien Haag, a shot sound estimated 1 meter away will regularly reports more intense in $\mathrm{dB}$ than cutting tools, jackhammers, and even a fly taking off 100 feet away.

Moreover, the "ascent time," or time from the beginning of the occasion to the primary pinnacle, is about momentary. One examination specifically discovered that the "gag impact," or unstable stun wave and sound vitality exuding from the weapon's barrel, frequently goes on for under 3 milliseconds.

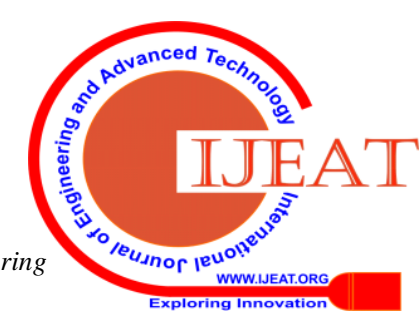




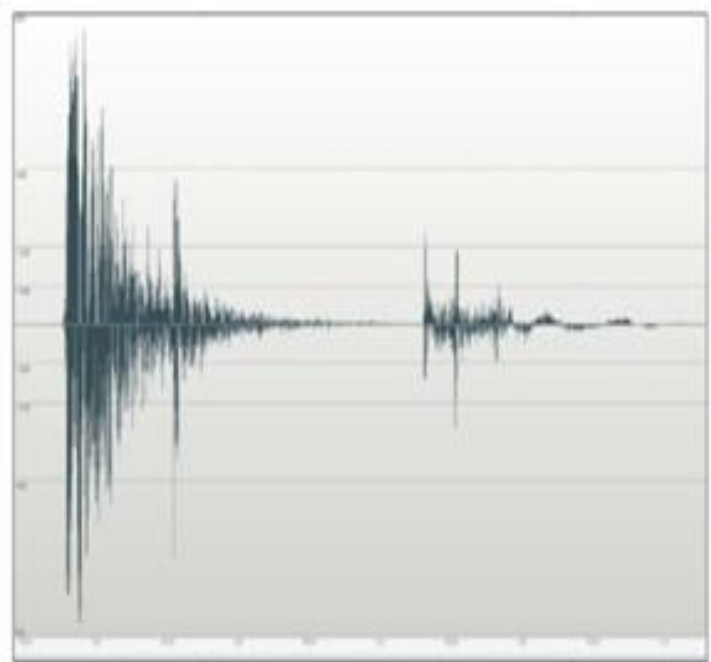

Fig-1. Waveform of 0.22 Caliber Rifle Gunshots with Reflection

This implies the shape and relative power of a discharge's "waveform," or visual portrayal of a sound sign or recording (used to show changes in adequacy after some time), can isolate a gunfire sound from others. Despite the fact that expenses and time allotments limit the materials utilized in this venture, these sound qualities can even now be saddled utilizing promptly accessible segments and natural programming. The waveform in Figure. 1 exhibits the essential qualities of a discharge sound, the high sign power and the close quick first crest from relative quietness. This account specifically was implied to have occurred at an outside terminating range. Notice an articulated reflection recorded in all respects rapidly after the starting occasion happens, in all probability the "reaction" of the first solid occasion ricocheting off the rearward holding divider or boundary used to quit approaching shots.

\subsection{Equipment And Configuration}

The framework starts with four mouthpieces. Amplifiers with high resistance to noisy driving forces would be perfect for a completely working framework utilized in a genuine task, however a reasonable pair of littler receivers is appropriate for this structure. The mouthpieces utilized in this test are electret condenser receivers. These are viewed as a stereo pair and all the four-catch sound all the while. Next, the mouthpieces are associated with a workstation phone an Arduino UNO link. This PC is outfitted with LABVIEW, which is an adaptable calculation and programming. LABVIEW handles both the sound info and the direction yield to the microcontroller. The real programming contents utilized in LABVIEW and with the microcontroller will be talked about later.

A microcontroller is then connected to the PC by means of sequential interface. For this situation, the association is by means of USB link. The microcontroller of decision is the Arduino Uno, because of its adaptability and broad open-source support. The Arduino acknowledges directions from the mouthpieces and sends a relating voltage to the framework.

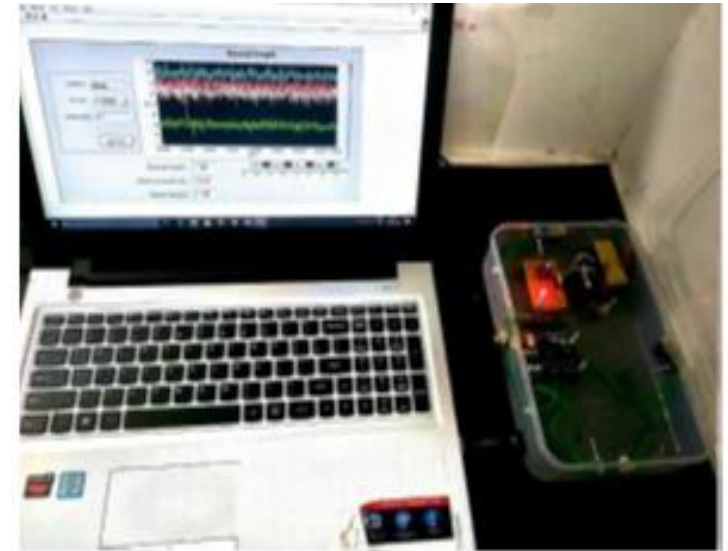

Figure-2 Basic Design of Gunshot Detection and Localization System.

Figure 2 is a photograph of the general perspective on the framework planned, gathered, and used for the testing sketched out in this venture. The amplifiers procure approaching sound and send to LABVIEW for handling. Should the approaching sign meet the limit prerequisites, LABVIEW would process the sign deferral and figure the heading. The Arduino gets the heading direction by means of sequential association.

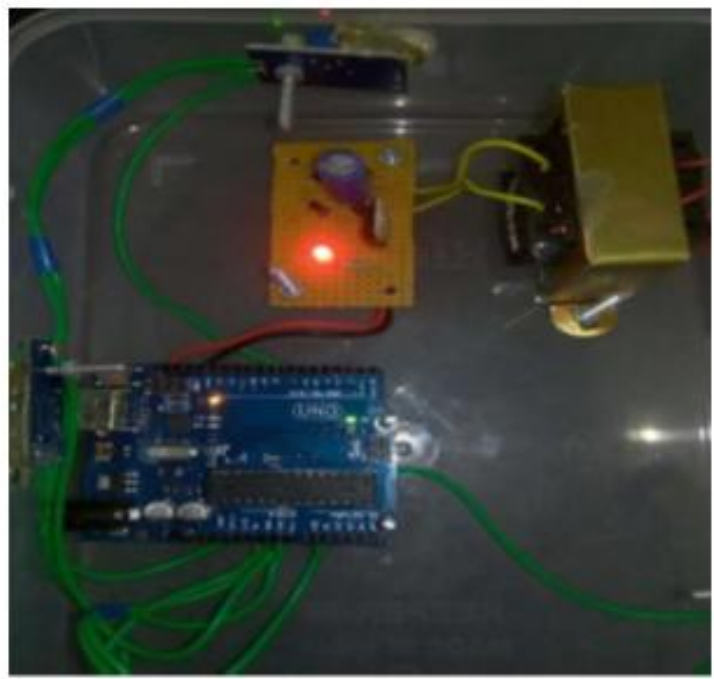

Figure-3 Arduino and Power Supply Set-up

A transformer alongside scaffold rectifier is utilized to change over air conditioning supply into dc. The scaffold rectifier is given voltage controller to balance out the dc voltage.

The Microphone setting is subject to different variables, including amplifier gain settings, anticipated that separation from sound source should receivers, and expected foundation clamor. Because of these numerous components, the setting requires cautious alignment for every sending. Accepting the speed of sound is roughly 350 meters for every second, a sound wave would travel 6 feet (or around 1.829 meters) in around .0053 seconds. 6 feet is the recommended separation between receivers utilized in the framework, .0053 seconds is the greatest deferral between channels. Since the examining recurrence characterized above is $48 \mathrm{kHz}$, or 48000 examples for each second, the

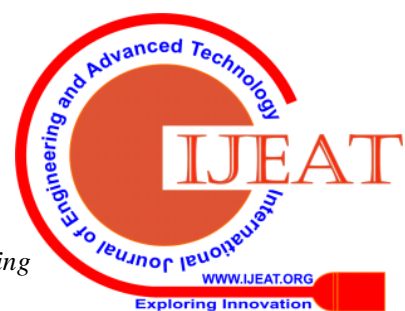


most extreme deferral between channels can likewise be estimated as around 255 examples.

\subsection{Programming}

With the framework arranged appropriately, the Arduino Arduino stage works fluidly with LabVIEW, enough so that the microcontroller can be modified to work persistently, hanging tight for LabView sequential directions, executing the directions, and returning outcomes whenever inquired. To set up the Arduino for this setup, the LabVIEW Support Package for Arduino (NI LabVIEW Interface for Arduino Toolkit) must be downloaded to the PC. Next a content must be composed for LabVIEW to consequently process the approaching sound and send directions likewise.

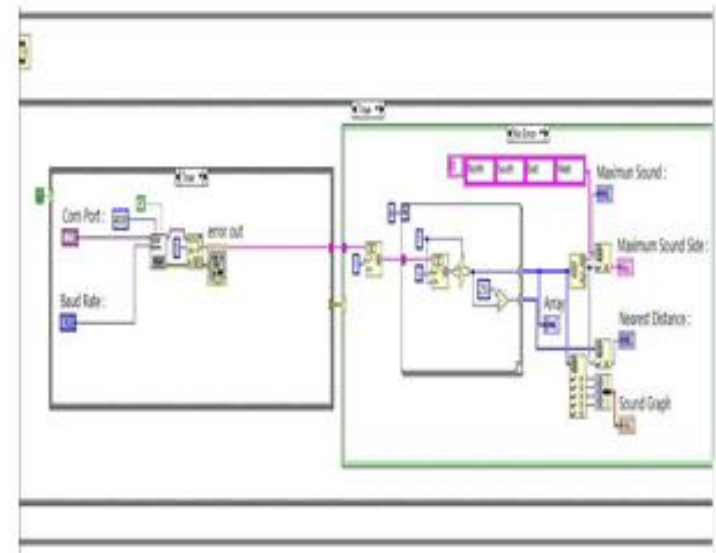

Figure-4. Overall Block Diagram of the System

Figure-4 speaks to the starting association of the Arduino UNO. The examining deferral is set by the required planning that one need the chart to be executed. Case structure is utilized for the general programming. Yield of the Com port is given to the VISA arrangement that has five bits designed in it. If there should be an occurrence of blunder yield will be seen in the exchange box close to the front board. At the point when the associations are precise another case structure is opened through a string subset. The string subset thusly circles to another to wipe out the primary byte and sending the staying four bytes for the procedure. Second subset string prompts pigeonhole so as to change over hexadecimal qualities into decimal. At that point the sort threw bytes are part and sent in two different ways. One to straightforwardly figure the separation by subtracting from 255 (Since 256 is the most extreme fs recurrence). Other one is wired to most extreme and least cluster. Pack idea is utilized so as to get synchronous charts of four unique headings. Chart portrayal is Waveform Data Type. Greatest Sound side is given four info strings to be specific North, East, South, West. Along these lines the general square chart part is run and the Front board presentations demonstrate the consequences of comparing bearings.

\section{TESTING AND RESULTS}

To assess the framework's usefulness, a straight forward test was detailed and executed. The framework was collected as portrayed above, in a progression of open, outside tennis court. This spot was picked with the goal of and LabVIEW should be modified appropriately. The

limiting potential meddling resonations, just as different factors presented in increasingly jam-packed zones. The test was executed during the evening, to diminish the opportunity of outer clamor impedance from wind or bystander. The air temperature was around 37 degrees Fahrenheit. This is huge in light of the fact that, albeit generally little changes in temperature would not influence the speed of sound in an extraordinary manner, it is outstanding that bigger temperature varieties could present complexities in computing the speed of sound.

As referenced before, the mouthpieces utilized were raised and divided at roughly 5 inches separated, with the idea that dispersing ought to be towards the more extensive end of the range to underline the postponement between approaching sound channels. Markers were set at 5, 10, and 15-foot separations from the middle point between amplifiers. Altogether, 3 markers were made. These markers showed the proposed positions from where the test sound would start.

At the season of the test, a genuine gun was not an accessible sound source. Rather, a noisy, sharp applaud of the hands was used at every marker. The general waveform state of a hand applaud could appropriately reproduce a discharge on the grounds that the two occasions can be described with high power and brief length. Despite the fact that the applauds were kept at predictable volume, some variety in sign force must be recognized. Notwithstanding, the varieties were viewed as satisfactory on account of the different components that present varieties in sound in a genuine circumstance. The test itself was intended to be controlled in most sensible viewpoints, yet took into account some similarity of a practical domain.

After the framework was amassed and started, the testing started. After each case of a hand applaud at every marker, the framework was assessed for a reaction and conceivable chart. The reactions were resolved after pinnacle signal in light of the applauds, or after a most extreme 5 applaud endeavors at the marker. Every marker was tried in a preliminary, with ten complete preliminaries making up the test.

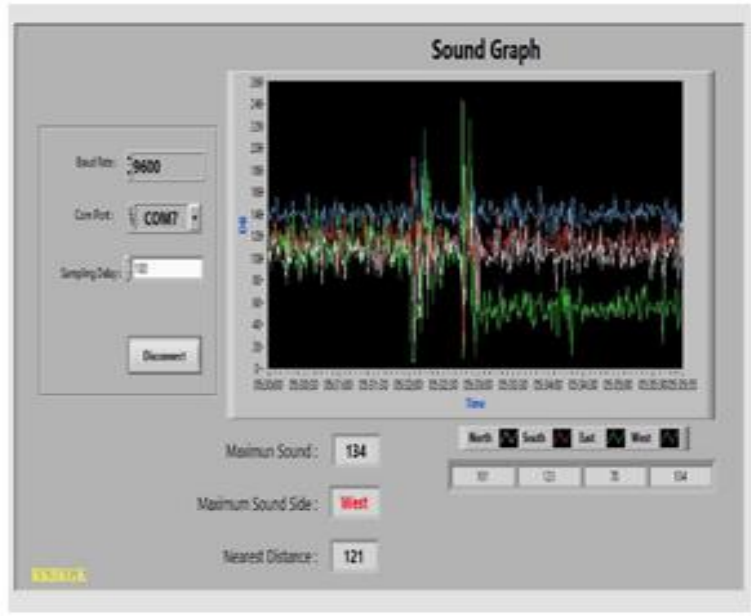

Figure-7. Graph Displaying All the Discriminating signals 


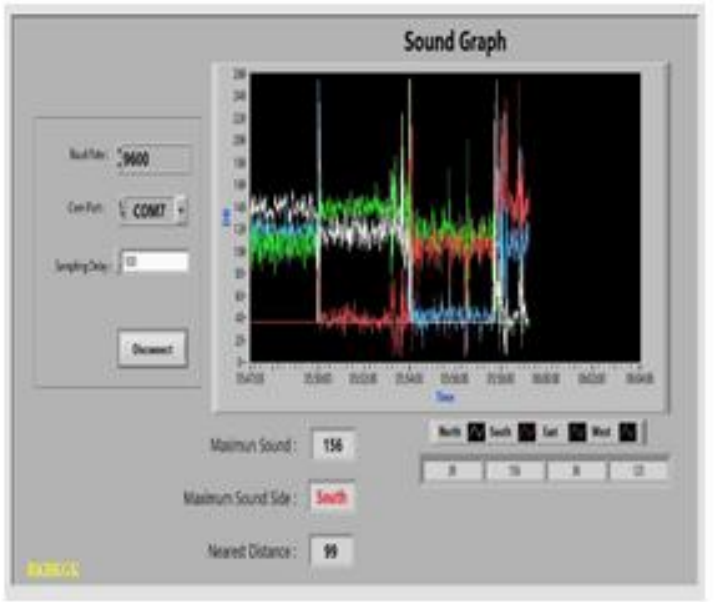

Figure-8. Graph representing North (White), East (Green) and South(Red) Direction as a Sharp Peak

Above fig 3.7 demonstrates the yield of the sound source as blended shading diagram where each chart speaks to every bearing. For example red shading speaks to south, white is for north, green and blue speaks to east and west individually. At the point when sound is from the specific bearing the expanding pinnacle shading demonstrates a similar course and shows in the most extreme sound side bed.

\subsection{Discussion}

At first look, the outcomes from the test overall appear to be blended and conflicting. To make the outcomes progressively applicable and really assess the test results for conflicting framework reactions, every preliminary was assessed as far as what number of "Possibly Useful Evidences" (or "PUEs") were made. These are characterized inside the domain of the test as chart reactions that end with the area of the sound source some place in edge, either focused or not focused. This is to reenact a certifiable situation, where a video recording of an episode would be submitted for proof. Conceivably valuable proof in such a situation would require the occasion itself or the prompt fallout to be caught some place in casing, either focused or not. Inside those parameters, the test came about with Table 3.1 .

\begin{tabular}{|c|c|c|c|c|c|c|c|c|c|}
\hline & $A$ & 5 & c & o & E & F & $\sigma$ & H & 1 \\
\hline 1 & Time - North & D6-North & ime-south & 8- Sosth & fine - East & D6- East & Time-west & se-west & \\
\hline 2 & 0530030 & 38 & Cs:30000 & 117 & $05330: 00$ & 32 & 0530000 & 36 & \\
\hline 3 & 0530001 & 134 & 05:30081 & 33 & C5:30001 & 138 & 053001 & 36 & \\
\hline 4 & $05: 30: 02$ & 124 & $05: 30002$ & 32 & 05300012 & 139 & $05=30.02$ & 36 & \\
\hline 5 & $05: 30: 03$ & 124 & Cs:3000] & 33 & 05330003 & 138 & 0530003 & 36 & \\
\hline 6 & $05: 30: 04$ & 115 & 05330084 & 32 & 05330054 & 139 & $05: 30: 04$ & 36 & \\
\hline 7 & 0530005 & 200 & C5:30005 & 38 & 053.3005 & 137 & 0530005 & 36 & \\
\hline 8 & C5:30:06 & 108 & $05: 30006$ & 32 & $05<30006$ & 145 & 05350.06 & 36 & \\
\hline 9 & $05: 30: 007$ & 217 & $05 \times 30007$ & 35 & 05300007 & 139 & 0530007 & 36 & \\
\hline 10 & $05: 30008$ & 99 & $05: 30008$ & 33 & $05: 30008$ & 155 & $05: 90: 08$ & 36 & \\
\hline iI & $05: 30009$ & 111 & $05: 30009$ & 32 & 05030009 & 136 & 0530009 & 36 & \\
\hline 12 & $00=30: 10$ & 125 & cos3c:10 & 32 & $05330: 10$ & 136 & $05=0010$ & 36 & \\
\hline 13 & $\cos 30: 11$ & 120 & $05: 3011$ & 34 & 00530211 & 136 & $05=30-11$ & 36 & \\
\hline 14 & $05: 30: 12$ & 113 & $05: 30: 12$ & 35 & $05: 30: 12$ & 200 & $05: 30-12$ & 115 & \\
\hline 15 & $\cos 30013$ & 137 & $\cos : 30: 13$ & 35 & $08: 30: 13$ & 203 & $05=30.13$ & 114 & \\
\hline 16 & $00530: 34$ & 138 & $05: 30: 34$ & 36 & $\cos : 30: 24$ & 304 & $05=30-14$ & 113 & \\
\hline 17 & $\cos 300.15$ & 138 & $\cos 30: 25$ & \% & 0530015 & 204 & 0530.15 & 113 & \\
\hline 19 & $05: 30: 16$ & 138 & $\cos 30: 16$ & 36 & $00330: 16$ & 209 & $05: 30-16$ & 113 & \\
\hline 19 & 0053017 & 138 & $00: 30: 17$ & 36 & CS:30:17 & 204 & $0530-17$ & 113 & \\
\hline 20 & Cosc30 18 & 139 & $\cos 300.28$ & 36 & $\cos : 30: 18$ & 204 & 053018 & 114 & \\
\hline 29 & $06: 30: 19$ & 139 & $00: 30: 19$ & 36 & $05: 30: 19$ & $=03$ & 0530019 & 113 & \\
\hline 22 & 0530020 & 138 & $05: 3020$ & 36 & $05: 3020$ & 203 & $05=30=20$ & 113 & \\
\hline 23 & $05: 302 n$ & 138 & $05: 30: 21$ & 36 & $05: 30221$ & 504 & 0530021 & 114 & \\
\hline
\end{tabular}

Table-1 Table Representing More Frequency of Sound in North Direction(Trial 1)
Table-2 Table Representing Max Frequency of Sound in West Direction(Trial 2)

\begin{tabular}{|c|c|c|c|c|c|c|c|c|c|}
\hline AI & - & $x \vee$ & f & fine- Nont & & & & & \\
\hline 4 & 2 & 1 & c & 0 & E & $F$ & 0 & H & 1 \\
\hline \multicolumn{2}{|c|}{1 time - North, } & \multicolumn{8}{|c|}{ 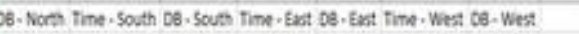 } \\
\hline 2 & $05: 30000^{\circ}$ & 215 & 0530000 & 114 & Cs:3000 & 56 & $05-30000$ & Ins & \\
\hline 3 & $0 \times 30001$ & 304 & 08302011 & 115 & es:sod: & 55 & Co: 30001 & 19 & \\
\hline 4 & 0039002 & m & 0530002 & 115 & $\operatorname{csson}$ & \$6 & $05: 3002$ & 139 & \\
\hline 5 & 00000003 & 103 & 0030003 & 124 & $\alpha=3003$ & s6 & 0530003 & 139 & \\
\hline 6 & $06: 30-04$ & 104 & $08: 30: 04$ & 114 & es:soted & 55 & 053004 & 500 & \\
\hline 7 & 05:30:05 & 104 & 053.30005 & 115 & os:00ess & 56 & 0530005 & Is & \\
\hline a & 0630006 & 304 & 0530006 & 215 & $\operatorname{css} 306$ & 57 & $05300 \%$ & 138 & \\
\hline ; & $0 \times 30007$ & 104 & 0830007 & 115 & Q5 30007 & $\$ 8$ & 083007 & 138 & \\
\hline 10 & 0500005 & 104 & 0830086 & 113 & $05: 30: 58$ & 58 & 0530008 & 138 & \\
\hline 11 & 0000000 & 105 & 0530000 & 115 & 6:50009 & 55 & 0530009 & 138 & \\
\hline 18 & 0630410 & 124 & 0533010 & 134 & c530:0 & 54 & 05301010 & 137 & \\
\hline 13 & 0030311 & 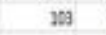 & $\cos 30011$ & 134 & es:son & $\$ 8$ & 0530011 & 139 & \\
\hline 14. & $0<: 3012$ & 204 & 053012 & 194 & 053012 & is & $05-30: 12$ & 13 & \\
\hline 15 & $05: 30: 13$ & ISS & 0530013 & 134 & o:s:10:13 & 56 & 0536013 & 1000 & \\
\hline 16. & cos:3014 & 103 & 0830044 & 194 & essop:24 & 56 & Cssen & Bg & \\
\hline 17 & 00530.25 & 193 & $0<30015$ & 194 & cs:so:ss & $\$$ & ossens & 139 & \\
\hline 13. & $08: 30416$ & 203 & $08: 30: 16$ & 134 & esso:2s & s8 & $05-30: 15$ & 139 & \\
\hline 19 & $06: 3017$ & 209 & 0630017 & 115 & C5:30:17 & 56 & $0530 \times 17$ & 199 & \\
\hline$x$ & 063018 & $1 m$ & 0530218 & 1115 & essasts & 56 & 053018 & 19 & \\
\hline 21 & $05 \times 3919$ & 203 & 0530019 & 124 & C5:30:59 & $\$ 6$ & 053019 & 139 & \\
\hline 22 & $0 \times 3020$ & 104 & 083020 & IIs & 053020 & 8 & $0 \times 3020$ & 139 & \\
\hline 23 & 0600231 & 103 & 0830211 & 114 & es:10:21 & 5s & 053021 & 139 & \\
\hline 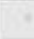 & 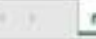 & mest tite mix & (4) & & & & & & \\
\hline
\end{tabular}

Above readings are legitimately traded from the sound chart of the LabVIEW front panel. The most extreme recurrence speaks to the bearing wherein sound is made. From this outcome it is clarified that sound inclined microphone gets most extreme power than the other three receivers.

\begin{tabular}{|c|c|c|c|c|c|c|c|c|c|}
\hline 4 & 4 & 5 & c & D & E & $f$ & G. & H & 1 \\
\hline [ & ine-North, & 6- North & 4. Sosth & - Sosch T & me-Ent o & 1. Eas T & se-West & 1. Wert & \\
\hline 2 & 0530000 & 103 & 0530000 & 113 & $05: 30,00$ & 36 & $05: 5000$ & 34 & \\
\hline 3 & 053001 & 36 & 0530001 & 2003 & 0800004 & 12 & Cs:30001 & 33 & \\
\hline 4 & es:30002 & $\%$ & CD 30002 & 1004 & $08: 3002$ & III & $05: 30002$ & 34 & \\
\hline 1 & $05: 30003$ & *6 & $05-30003$ & 104 & Co:30013 & 114 & CS:s0es & 3 & \\
\hline 6 & 0506004 & 112 & 050004 & 35 & 0600004 & 139 & $05: 3004$ & 36 & \\
\hline 3 & s.sens & 244 & cosuss & 16 & t6:000s & wo & Cs:anes & 16 & \\
\hline 8 & S530:06 & 24s & 0530006 & 35 & 0509096 & tas & 050000 & 36 & \\
\hline 9 & $0: 30007$ & 133 & Q5:30:07 & 35 & $06: 90007$ & 199 & C5:30s] & 36 & \\
\hline 10 & 0530008 & II3 & $0 \times 3008$ & $\%$ & 0503008 & 199 & $05: 0008$ & 35 & \\
\hline 11 & $05: 3000$ & 14 & QS:30009 & 35 & $08: 30: 09$ & 138 & CS::0009 & 36 & \\
\hline 12. & $0530: 10$ & 14 & Q5:30:10 & 35 & $\cos 230: 20$ & 139 & $05: 300.00$ & 36 & \\
\hline 13 & es:30:11 & 13 & $\theta=30: 11$ & 3s & $08: 30: 31$ & 139 & $c s: s a n$ & 36 & \\
\hline 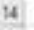 & osto:12 & 124 & $0530: 12$ & is & $06: 00: 12$ & 158 & $C 5: 012$ & 36 & \\
\hline 15. & 00001 & ws & (5acts & $x$ & c5:s:al] & 288 & C5:16:13 & 15 & \\
\hline 16 & es:-10:14 & m & es:at: & s & $60: 253$ & 138 & 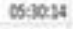 & 36 & \\
\hline 17 & 053015 & 24 & Q5 30.15 & 36 & $05: 0015$ & 29 & Co:30:15 & 36 & \\
\hline 18 & S53016 & $m$ & es30:16 & 34 & $05: 30: 36$ & 139 & $05: 50: 25$ & 36 & \\
\hline 19 & $0530: 17$ & 113 & $0530: 17$ & 30 & $08: 3017$ & 237 & $05: 00: 17$ & 36 & \\
\hline 20 & es:30118 & 124 & 0530018 & 33 & $08: 30: 18$ & 138 & $05: 30: 8 s$ & 36 & \\
\hline 21 & os $30: 19$ & 134 & es $300: 19$ & 33 & $06: 30: 19$ & 139 & 0530.19 & 36 & \\
\hline$n$ & $\cos 30020$ & in & $\theta=30 x 0$ & 33 & $\cos 000$ & 138 & $\cos 3020$ & 36 & \\
\hline a) & $\cos 100 \pi t$ & 244 & csacent & us & c6:10:22 & is & $\cos 10021$ & $\mathbf{B}$ & \\
\hline
\end{tabular}

Table-3 Table Representing Maximum Frequency of Sound in East Direction (Trial 3)

Presently by performing back to back trails a table is gotten that demonstrates the PUE results. Table. 3 Test Trial Potentially Useful For Evidence Results. This is an illogical outcome, since one would accept that occasions at further separations are bound to occur inside the casing of view.

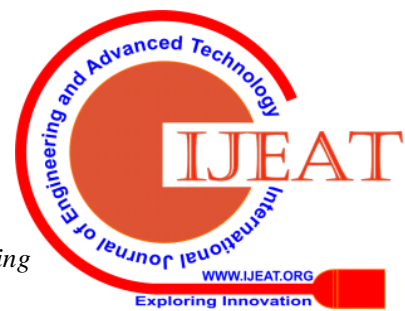


The general irregularity in the framework can be credited to various variables. As a matter of first importance, the amplifiers utilized may not be most reasonable for the errand of separation. Because of the brief span of most shot sounds, receivers utilized should be touchy enough to precisely characterize the approaching sound data, to the point wherein a progression of noisy driving forces divided firmly together in quick progression would be recorded thusly, rather than one long, boisterous motivation. The microphones utilized in this test were not explicitly planned in light of that task.

Second, the encompassing condition assumes an enormous job in the viability of these frameworks. Despite the fact that the testing area and time of day were picked with the plan that wild factors would be limited, not all things potentially be represented, and minor changes in the test conditions will make variances in information.

At long last, the programming language utilized in LABVIEW itself could utilize some survey and potential overhauling. So as to infer the right edges at a specific setting, testing must be done to decide a mix that works best. A dimension of computerization for limits may be deserving of some consideration, where the framework could be intended to consequently alter for changes in the commotion floor, and so forth.

Continuous versatile channels may likewise work as far as constraining the measure of incidental and futile sound data that would just block advance, particularly hints of frequencies underneath around $400 \mathrm{~Hz}$ and more prominent than $2.5 \mathrm{kHz}$, which are the frequencies shown by gunfire.

These guidelines, tests, and results are helpful in depicting the procedure for fundamental discovery and reaction, yet the plan carries a hidden defect. This is on the grounds that further separation of discharge sounds from different clamors of a similar shape includes more elevated amount sifting and investigation of the sounds, by means of the programming. Shockingly, shot sound as a rule shows tops around $630 \mathrm{~Hz}$.

These reaches are basic for a wide assortment of different sounds, making segregation considerably more convoluted.

In Figure-1. The frequencies of most astounding force go from the least up to $2 \mathrm{kHz}$, with greatest qualities underneath $500 \mathrm{~Hz}$. These outcomes do consolidate some "ecological acoustics" because of the appearance in the chronicle, however that would be normal for most sound occasions in reasonable situations.

To appropriately separate from different sounds, the best methodology now is an algorithmic learning procedure, for example, those proposed by Morton and Collins or Valenzise et al. To appropriately mimic the items utilized in the field and endeavor to recreate their usefulness, merchants, for example, Shot Spotter ought to furnish researchers with certain information identified with their item determinations and schematics and for research purposes.

This contentions with the merchants' rights to retain restrictive data, yet more critically energizes fair audit and testing in a logical and friend surveyed discussion. Those sorts of methods would help lighten questions about these frameworks' capacities, and may improve their overall population picture, as saw beneath. At the season of this test, delegates from both Shot Spotter and Boomerang chose not for field inquiries regarding explicit components of their particular frameworks' plans, functionalities, and test information.

\section{REFERENCES}

1. Bales W, Mann K, Blomberg T, Gaes G, Barrick K, et al. (2010) A Quantitative and Qualitative Assessment of Electronic Monitoring. Washington, D.C.: National Institute of Justice. $208 \mathrm{p}$.

2. Haag MG, Haag LC.,(2011) Shooting Incident Reconstruction. 2nd rev. ed. San Diego: Academic Press.

3. Mares D, Blackburn E (2012) Evaluating the Effectiveness of an Acoustic Gunshot Location System in St. Louis, MO. Policing 6: 26-42

4. Monisa, S., Vijayachitra, S., 2015, "Real Time Performance Analysis and Fault Diagnosis in Heat Exchanger", International Journal of Chemtech Research, Vol.8, No.12, pp.418-427

5. Sallai J, Hedgecock W, Volgyesi P, Nadas A, Balogh G, et al. (2011) Weapon classification and shooter localization using distributed multichannel acoustic sensors. Journal of Systems Architecture 57: 869-885

6. Ramos, A. L. L., Holm, S., Gudvangen, S., \& Otterlei, R.(2011, May). Delay-and-sum beamforming for direction of arrival estimation applied to gunshot acoustics. In Proc. spie, sensors, and command, control, communications, and intelligence (c3i) technologies for homeland security and homeland Défense x (Vol. 8019, p. 80190U 1-9).

7. Ramos, A. L. L., Holm, S., Gudvangen, S., \& Otterlei, R. (2012, May). Real-time vehicle noise cancellation techniques for gunshot acoustics. In Proc. spie, sensors, and command, control, communications, and intelligence (c3i) technologies for homeland security and homeland defense xi (Vol. 8359, p. 835917- 835917-9).

8. Smith EL, Cooper A (2013) Homicide in the U.S. Known to Law Enforcement, 2011. Washington, D.C.: U.S. Department of Justice Bureau of Justice Statistics.

9. Thornton RL, Burrell WD, Dunlap KL, Schweer RG (2009) Guns, Safety and Proactive Supervision: Involving Probation and Parole in Project Safe Neighborhoods. Washington, D.C.: U.S. Department of Justice Bureau of Justice Assistance. $56 \mathrm{p}$.

10. Yuvarani P, 2012, 'Image denoising and enhancement for lung cancer detection using soft computing technique,' IET Chennai 3rd International on Sustainable Energy and Intelligent Systems (SEISCON 2012), Tiruchengode, pp. 1-4. doi: 10.1049/cp.2012.2179.

11. Zhang, B., Liu, H., Chen, F., \& Wang, G. (2012, July) Numerical simulation of flow fields induced by a supersonic projectile moving in tubes. Shock Waves,22, 417-425. doi: 10.1007/s00193-012-0389-4 